PROCEEDINGS OF THE

AMERICAN MATHEMATICAL SOCIETY

Volume 130, Number 10, Pages 2857-2864

S 0002-9939(02)06460-2

Article electronically published on March 25, 2002

\title{
A PROOF OF A PARTITION THEOREM FOR $[\mathbb{Q}]^{n}$
}

\author{
VOJKAN VUKSANOVIC
}

(Communicated by Alan Dow)

\begin{abstract}
In this note we give a proof of Devlin's theorem via Milliken's theorem about weakly embedded subtrees of the complete binary tree $2^{<\mathbb{N}}$. Unlike the original proof which is (still unpublished) long and uses the language of category theory, our proof is short and uses direct combinatorial reasoning.
\end{abstract}

A tree is any partially ordered set $(T, \leq)$ such that for any $t \in T$ the set $\{s \in$ $T: s \leq t\}$ is well ordered by the induced order. Given a tree $T$ we say that $S$ is a subtree of $T$ if $S \subseteq T$. From now on we will suppose that every tree has a root, i.e. we will suppose that every tree has the unique minimal element. We will also suppose that that for a given tree $T$, for every $t \in T,\{s \in T: s \leq t\}$ is finite. By $\operatorname{Succ}(t, T)$ we will denote the set $\{s \in T: t \leq s\}$ and by $\operatorname{Pred}(t, T)$ we will denote the set $\{s \in T: s \leq t\}$. Given a tree $T$ and $t \in T$ by $I S(t, T)$ we will denote the set of immediate succesors of $t$ in $T$. By $T(n)$ we will denote the $n$-th level of $T$, i.e. the set of all $t \in T$ such that $|\{s \in T: s \leq t, s \neq t\}|=n$. For a tree $T$, height $(T)$ will denote $\sup \{n+1: T(n) \neq \emptyset\}$. We say that a tree $(T, \leq)$ is perfect if for every $t \in T$ there is $s \in T$ such that $t \leq s$ and $|I S(s, T)| \geq 2$. Given a tree $T$ and nodes $s$ and $t$ in $T$ by $s \wedge t$ we will denote the maximal node in $T$ which is below both $s$ and $t$. If $A \subseteq T$, by $\wedge(A)$ we will denote the $\wedge$ - closure of $A$, the smallest subset $A^{\prime}$ of $T$ containing $A$ such that $\left(\forall s, t \in A^{\prime}\right) s \wedge t$ belongs to $A^{\prime}$.

Most of the time we shall be working inside the complete binary tree $2^{<\mathbb{N}}$ ordered by end-extension which we denote by $\subseteq$. We shall also need to consider the two orderings on $2^{<\mathbb{N}},<_{l e x}$ and $\leq_{\mathbb{Q}}$, which we introduce in the following two definitions.

Definition 0.1. Let $s, t \in 2^{<\mathbb{N}}$. We say that $s$ is lexicographically less than $t$ and write $s<_{\text {lex }} t$ provided the following hold: $s$ and $t$ are incomparable and if $i \in \mathbb{N}$ is the maximal integer such that $s\lceil i+1=t\lceil i+1$, then $s(i+1)<t(i+1)$.

Definition 0.2. Given $s, t \in 2^{<\mathbb{N}}$ put $t \leq \mathbb{Q} s$ iff $s=t$ or $\left(\left(s \subseteq t\right.\right.$ and $\left.s^{\wedge} 0 \subseteq t\right)$ or $\left(t \subseteq s\right.$ and $\left.t^{\wedge} 1 \subseteq s\right)$ or $(s$ and $t$ are incomparable and $t<$ lex $\left.s)\right)$.

Note that while $\leq_{\mathbb{Q}}$ is a total order $<_{l e x}$ is not. But, $<_{l e x}$ is a total order on any antichain of $2^{<\mathbb{N}}$ and in fact it agrees with $\leq_{\mathbb{Q}}$. It is also easy to check that $\left(2^{<\mathbb{N}}, \leq_{\mathbb{Q}}\right)$ has the order type $\eta$.

Let $A \in\left[2^{<\mathbb{N}}\right]^{n}$ for some $n \in \mathbb{N}$. From now on by $\left\{a_{1}, \ldots, a_{n}\right\}_{\leq_{Q}}$ we will denote the enumeration of elements of $A$ with respect to $\leq_{\mathbb{Q}}$.

Received by the editors March 29, 2001 and, in revised form, May 29, 2001.

2000 Mathematics Subject Classification. Primary 05A18.

Key words and phrases. Partitions of rationals. 


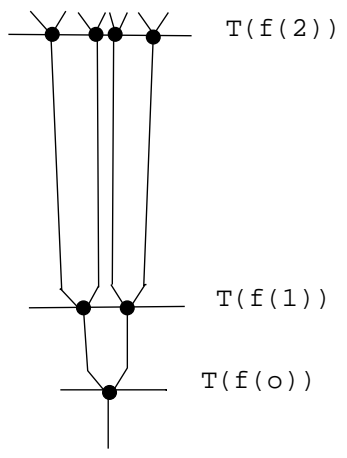

FiguRE 1.

Definition 0.3. Suppose that $S$ is a subtree of $T$. We say that $S$ is strongly embedded in $T$ (see Figure 1 for the case $T=2^{<\mathbb{N}}$ ) if the following hold:

1) If $s$ is a nonmaximal in $S$ and $t \in I S(s, T)$, then $\operatorname{Succ}(t, T) \cap I S(s, S)$ is a singleton,

2) If height $(T)=\omega$ and height $(S) \leq \omega$ there is strictly increasing function $f:$ height $(S) \rightarrow \omega$ such that $S(n) \subseteq T(f(n))$ for each $n \in$ height $(S)$.

Suppose in the previous definition we drop requirement 2), and instead of 1) we have:

$\left.1^{\prime}\right)$ If $s$ is a nonmaximal in $S$ and $t \in I S(s, T)$, then $\operatorname{Succ}(t, T) \cap I S(s, S)$ is either a singleton or empty.

Then we say that $S$ is weakly embedded in $T$. $T$.

By $W E m^{<\omega}(T)$ we will denote the set of all finite weakly embedded subtrees of

Definition 0.4. Given a strongly embedded subtree $T$ of $2^{<\mathbb{N}}$ and trees $A$ and $B$ weakly embedded in $T$, we say that $A$ and $B$ have the same embedding type and we write $A \sim_{E m} B$ provided the following hold:

1) There is a bijection $f: A \rightarrow B$ satisfying $a \subseteq a^{\prime}$ iff $f(a) \subseteq f\left(a^{\prime}\right)$.

2) If $a \in A \cap T(n), a^{\prime} \in A \cap T\left(n^{\prime}\right), f(a) \in B \cap T(m)$, and $f\left(a^{\prime}\right) \in B \cap T\left(m^{\prime}\right)$, then $n<n^{\prime}$ iff $m<m^{\prime}$.

3) Suppose $n \in \omega$ and there is $d \in A \cap T(n)$. Suppose $a \in T(n)$ with $(\operatorname{Succ}(a, T) \backslash$ $\{a\}) \cap A \neq \emptyset$. Pick $c \in(\operatorname{Succ}(a, T) \backslash\{a\}) \cap A$, and let $b$ be the unique node with $b \in \operatorname{Pred}(c, T) \cap T(n+1)$. Suppose $f(d) \in T(m)$, and write $a^{\prime}$ and $b^{\prime}$ for the unique nodes $a^{\prime} \in(\operatorname{Pred}(f(c), T) \backslash\{f(c)\}) \cap T(m)$ and $b^{\prime} \in \operatorname{Pred}(f(c), T) \cap T(m+1)$. Then we require that $a^{\wedge} 0 \subseteq b$ iff $a^{\prime \wedge} 0 \subseteq b^{\prime}$ (see Figure 2).

Note that this is not the same as the original definition given in $[3$. Originally, we have that in a finitely branching tree $T$, for each $t \in T$ there is a linear order $\prec$ on $I S(t, T)$. In other words, for each $t \in T, I S(t, T)$ can be enumerated as

$$
I S(t, T)=\{i s(t, T)(j): j \in|I S(t, T)|\}
$$

so that

$$
i s(t, T)(i) \prec i s(t, T)(j) \Leftrightarrow i \in j \in \mid I S t, T) \mid .
$$



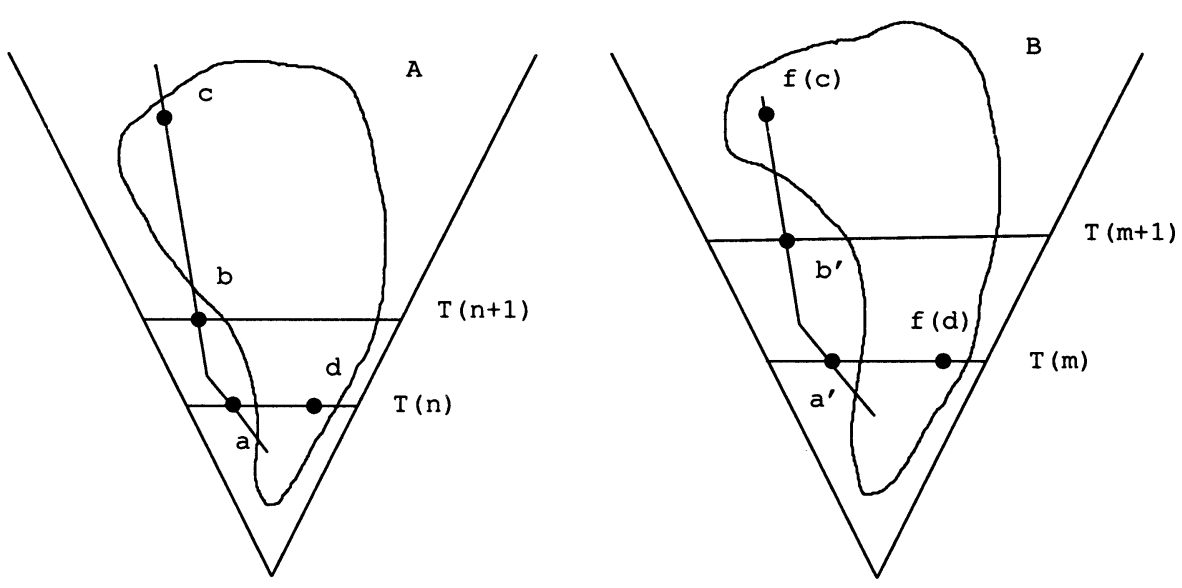

FiguRE 2.

Also, in 3) of the previous definition of the embedding type of a weakly embedded subtree, it is required that for each $i \in|I s(a, T)|, b=i s(a, T)(i)$ iff $b^{\prime}=i s\left(a^{\prime}, T\right)(i)$. In the case of strongly embedded subtrees of the complete binary tree we naturally take the ordering $<_{\text {lex }}$ to be the linear order $\prec$. Then it is easy to see that our definition is equivalent to the original one when working with strongly embedded subtrees of $2^{<\mathbb{N}}$. We write $\operatorname{Em}^{A}(T)$ for the collection of all weakly embedded subtrees $B$ of $T$ with $A \sim_{E m} B$. The following theorem, which is due to Milliken [3], is crucial for our proof.

Theorem 0.5. Suppose that $T$ is a perfect strongly embedded subtree of $2^{<\mathbb{N}}$, that $A \in W \operatorname{Em}^{<\omega}(T)$, and that $\operatorname{Em}^{A}(T)=\bigcup_{i \in r} C_{i}$. Then there is a perfect strongly embedded subtree $S$ of $T$ and $k \in r$ such that $\operatorname{Em}^{A}(T) \cap W E m^{<\omega}(S) \subseteq C_{k}$.

We will now state the theorem which is due to Devlin [2].

Theorem 0.6. We have:

1) $\quad \eta \rightarrow(\eta)_{<\omega / t_{n}}^{n}$,

2) $\quad \eta \nrightarrow(\eta)_{<\omega / t_{n}-1}^{n}$ where $t_{1}=1$ and $t_{n}=\sum_{i=1}^{n-1}\left(\begin{array}{c}2 n-2 \\ 2 i-1\end{array}\right) t_{i} t_{n-i}$ for $n \geq 2$.

We will prove three lemmas which will prove Theorem 0.6. The first two prove 1 ), and the last proves 2 ).

From now on we identify the rationals with the set of all finite sequences of 0 's and 1's.

Given a colouring $c:\left[2^{<\mathbb{N}}\right]^{n} \rightarrow r$, it naturally induces a colouring $c^{\prime}: \mathcal{F} \rightarrow r$ where $\mathcal{F}$ is a family of all subsets $A$ of $2^{<\mathbb{N}}$ such that there is an $A^{\prime} \in\left[2^{<\mathbb{N}}\right]^{n}$ and $A=\wedge\left(A^{\prime}\right)$. It is easy to see that we have only finitely many different embedding types which appear as a $\wedge$ - closure of $n$-element subsets of $2^{<\mathbb{N}}$. For example the $\wedge$ - closure of a pair in $2^{<\mathbb{N}}$ gives us seven different embedding types. These are (see Figure 3) $\{\{\emptyset, 0\},\{\emptyset, 1\},\{\emptyset, 0,1\},\{\emptyset, 0,10\},\{\emptyset, 0,11\},\{\emptyset, 1,00\},\{\emptyset, 1,01\}\}$.

Hence, we can apply Theorem 0.5 successively finitely many times to get a perfect strongly embedded subtree $T$ of the complete binary tree $2^{<\mathbb{N}}$ such that if $A=$ 


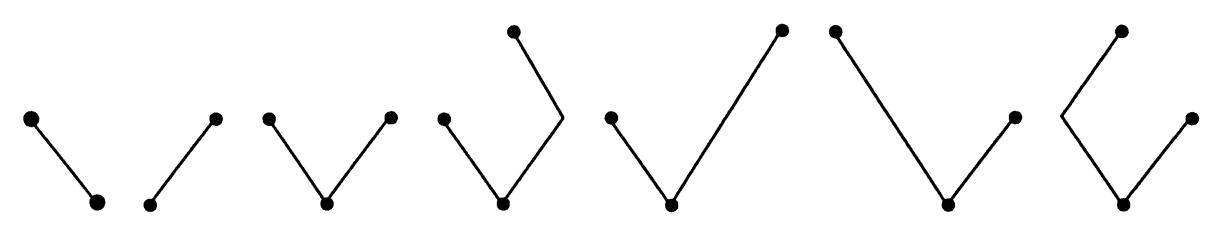

FiguRE 3.

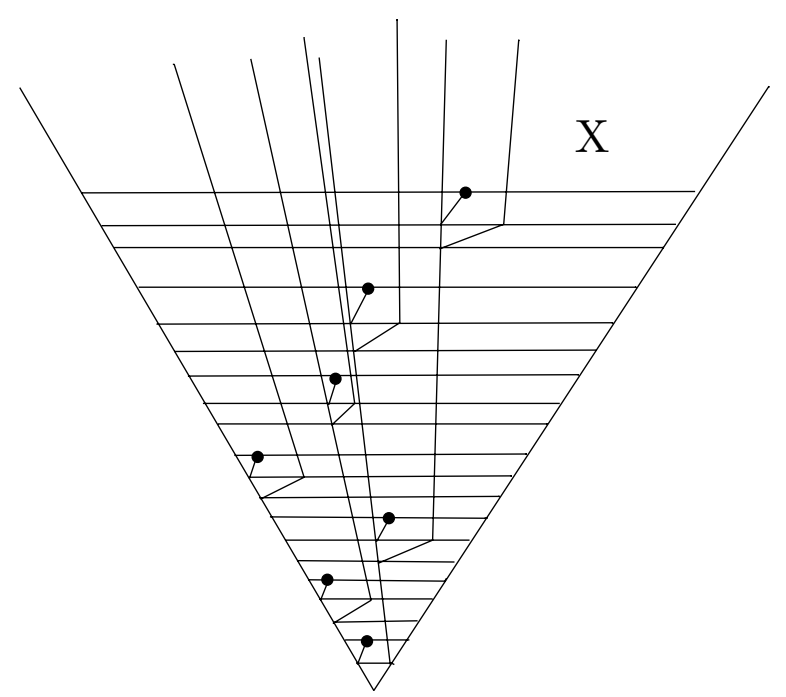

FIGURE 4.

$\left\{a_{1}, a_{2}, \ldots, a_{n}\right\}$ and $B=\left\{b_{1}, b_{2}, \ldots, b_{n}\right\}$ are two different $n$-element subsets of $T$ and if $\wedge(A) \sim_{E m} \wedge(B)$, then $c(A)=c(B)$. We can assume without loss of generality that we have the complete binary tree $2^{<\mathbb{N}}$ such that all $n$-element subsets which have $\wedge$ - closure of the same embedding type are monochromatic.

It turns out that there is a subset $X$ of $2^{<\mathbb{N}}$ large enough in the sense that it has order type $\eta$ under $<_{l e x}$ and which has the property that for each $n$ the members of $[X]^{n}$ realise the minimal possible number of embedding types over all subsets of $2^{<\mathbb{N}}$ of order type $\eta$ under $\leq_{\mathbb{Q}}$. We introduce the set $X$ in the following definition.

Definition 0.7. Let $\left\{e_{n}: n \in \mathbb{N}\right\}$ be an enumeration of the complete binary tree with the property that for any two $e_{n}, e_{m}$ we have $m<n$ iff $\left|e_{m}\right|<\left|e_{n}\right|$ or $\left(\left|e_{m}\right|=\left|e_{n}\right|\right.$ and $\left.e_{m}<_{l e x} e_{n}\right)$. Define $\phi: 2^{<\mathbb{N}} \rightarrow 2^{<\mathbb{N}}$ by induction as follows: $\phi(\emptyset)=\emptyset$ and given $\phi(t)$ if $t^{\wedge} i=e_{n}$ put $\phi\left(t^{\wedge} i\right)=\phi(t)^{\wedge} i^{\wedge} 00 \ldots 0$ and $\left|\phi\left(t^{\wedge} i\right)\right|=3 n$, where $i=0,1$. From now on by $W$ and $X$ we will denote the following two sets, $W=\phi^{\prime \prime} 2^{<\mathbb{N}}$ and $X=\left\{\phi(t)^{\wedge} 01: t \in 2^{<\mathbb{N}}\right\}$ (see Figure 4).

Note that $X$ is an antichain and that $\left(X,<_{l e x}\right)=\left(X \leq_{\mathbb{Q}}\right)$ has the order type $\eta$. Let $A \in[X]^{n}$ be arbitrary. It is easy to see that $|\wedge(A)|=2 n-1$. It is also easy to see that for any $Y \subseteq X$ we have $\wedge(Y)=\{s \wedge t: s, t \in Y\}$. Let $\wedge(A)=\left\{a_{1}, a_{2}, \ldots, a_{2 n-1}\right\}_{\leq_{\mathbb{Q}}}$. Define a well-ordering $<_{W_{A}}$ of the set $\{1,2, \ldots, 2 n-1\}$ as follows: $i<W_{A} j$ iff $\left|a_{i}\right|<\left|a_{j}\right|$. Then we have the following lemma. 
Lemma 0.8. Let $A$ and $B$ be two n-element subsets of $X$. If $\wedge(A) \sim_{E m} \wedge(B)$, then $<_{W_{A}}=<_{W_{B}}$.

Proof. We will prove this by induction on $n$. For $n=1$ the lemma holds trivially. Suppose that the lemma is true for every $k \leq n$ and let us prove the lemma for $n+1$. Let $A, B \in[X]^{n+1}$ and let $A^{\prime}=\left\{a_{1}, a_{2}, \ldots, a_{2 n+1}\right\}_{\leq_{\mathbb{Q}}}$ and $B^{\prime}=\left\{b_{1}, b_{2}, \ldots, b_{2 n+1}\right\}_{\leq_{\mathbb{Q}}}$ where $A^{\prime}=\wedge(A)$ and $B^{\prime}=\wedge(B)$. Let $a_{k}$ and $b_{l}$ be the roots of $A^{\prime}$ and $B^{\prime}$ respectively. Set $A_{1}=\left\{x \in A^{\prime}: a_{k}{ }^{\wedge} 0 \subseteq x\right\}, A_{2}=\left\{x \in A^{\prime}: a_{k}{ }^{\wedge} 1 \subseteq x\right\}$ and similarly define $B_{1}$ and $B_{2}$. Then, if $f: A^{\prime} \rightarrow B^{\prime}$ is a bijection witnessing $A^{\prime} \sim_{E m} B^{\prime}$ it is easy to see that $f\left(a_{k}\right)=f\left(b_{l}\right)$ and therefore $k=l$. Also, it is easy to see that $f\left\lceil A_{1}\right.$ is a bijection from $A_{1}$ onto $B_{1}$ witnessing $A_{1} \sim_{E m} B_{1}$ and $f \uparrow A_{2}$ is a bijection from $A_{2}$ onto $B_{2}$ witnessing $A_{2} \sim_{E m} B_{2}$. Therefore, we can apply inductive hypothesis to $A_{1}, B_{1}$ and to $A_{2}$ and $B_{2}$ and easily conclude the lemma.

Hence, from the lemma above one concludes that the embedding type of the $\wedge(A)$ of some $A \in[X]^{n}$ is determined completely by its well-ordering $<_{W_{A}}$. This will help us to count the number of possible colours for $n$-tuples of $X$.

Lemma 0.9. For each $n$ let $t_{n}$ be the minimal number of embedding types which appear as the $\wedge$-closure of $n$-element subsets of $X$. Then

$$
t_{1}=1 \quad \text { and } \quad t_{n}=\sum_{i=1}^{n-1}\left(\begin{array}{c}
2 n-2 \\
2 i-1
\end{array}\right) t_{i} t_{n-i} .
$$

Proof. That $t_{1}=1$ is easy and well-known. So, suppose that $n>1$ and let $A^{\prime}$ be the $\wedge$ - closure of some $n$-element subset $A=\left\{a_{1}, a_{2}, \ldots, a_{n}\right\}$ in $X$. Set $B^{\prime}=\left\{x \in A^{\prime}: a^{\wedge} 0 \subseteq x\right\}$ and $C^{\prime}=\left\{x \in A^{\prime}: a^{\wedge} 1 \subseteq x\right\}$ where $a$ is the root of $A^{\prime}$. Let $l=\left|\left\{a_{i}: a_{i} \in B^{\prime}\right\}\right|$ and $r=\left|\left\{a_{i}: a_{i} \in C^{\prime}\right\}\right|$. Note that $1 \leq l, r$ and $l+r=n$. Note also that $B^{\prime}=\wedge(B)$ and $C^{\prime}=\wedge(C)$ where $B=A \cap B^{\prime}$ and $C=A \cap C^{\prime}$. Note that for fixed $l, r \leq n$ we have by inductive hypothesis $t_{l} t_{r}$ different types of pairs $\langle B, C\rangle$. By the previous lemma structure $A^{\prime}$ is completely determined by its well-ordering $<_{W_{A}}$. The well-ordering $<_{W_{A}}$ interlaces the well-orderings $<_{W_{B}}$ and $<_{W_{C}}$ and adjoins $a$ as the $<_{W_{A}}$ minimal element. (For $n=2$ and $n=3$ see Figure 6 and Figure 7 respectively.) The number of possible ways $<_{W_{B}}$ and $<_{W_{C}}$ can be interlaced to form $<_{W_{A}}$ is $\left(\begin{array}{c}\left|B^{\prime}\right|+\left|C^{\prime}\right| \\ \left|B^{\prime}\right|\end{array}\right)=\left(\begin{array}{c}2 n-2 \\ 2 l-1\end{array}\right)$. Therefore, the total number of possibilities for $A^{\prime}$ is

$$
t_{n}=\sum_{l=1}^{n-1}\left(\begin{array}{c}
2 n-2 \\
2 l-1
\end{array}\right) t_{l} t_{n-l}
$$

This proves 1) of Devlin's theorem. The next lemma will prove 2).

Lemma 0.10. Let $S \subseteq X$ and let $S$ have $\leq_{\mathbb{Q}}$ - order type $\eta$. Then for every $P \in[X]^{n}$ there is $A \in[S]^{n}$ such that $\wedge(P) \sim_{E m} \wedge(A)$.

Proof. Set $S^{\prime}=\left\{x \in W\right.$ : both $\left\{s \in S: x^{\wedge} 0 \subseteq s\right\}$ and $\left\{s \in S: x^{\wedge} 1 \subseteq s\right\}$ are densely ordered by $\left.\leq_{\mathbb{Q}}\right\}$. We will prove that $S^{\prime}$ is a perfect subtree. Let $s^{\prime}$ be the root of the $\wedge(S)$. Note that $s^{\prime} \in W$. Put $U=\left\{t \in S: s^{\prime \wedge} 0 \subseteq t\right\}$ and $V=\left\{t \in S: s^{\prime \wedge} 1 \subseteq t\right\}$. Then $U$ and $V$ are nonempty, $U \cup V=S$ and $\forall u \in U$ and $\forall v \in V$ we have that $u \leq_{\mathbb{Q}} v$. Since $S$ has order type $\eta, U$ and $V$ are densely ordered by $\leq_{\mathbb{Q}}$ and $s^{\prime} \in S^{\prime}$. Given $s \in S^{\prime}$ put $U=\left\{t \in S: s^{\wedge} 0 \subseteq t\right\}$ and let $U^{\prime}=U \backslash\{u \in U: u$ is an 


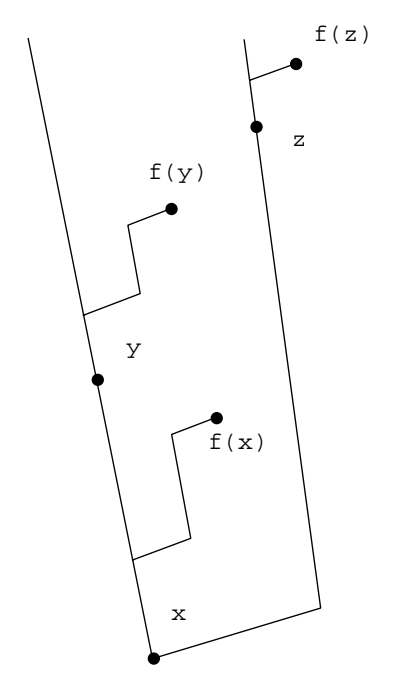

Figure 5.
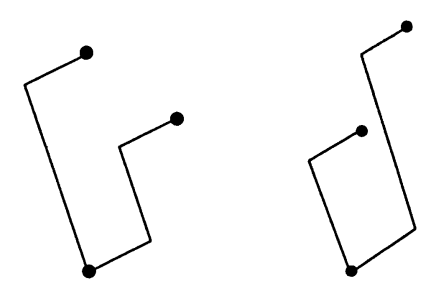

Figure $6 . \quad$ Embedding types in the case $n=2$

endpoint of the dense linear order $\left.\left(U, \leq_{\mathbb{Q}}\right)\right\}$. Let $u$ be the $\subseteq$ - least node of the $\wedge\left(U^{\prime}\right)$. As above, both $\left\{w \in U^{\prime}: u^{\wedge} 0 \subseteq w\right\}$ and $\left\{w \in U^{\prime}: u^{\wedge} 1 \subseteq w\right\}$ are densely ordered by $\leq \mathbb{Q}$. Because the only points deleted from $U$ to form $U^{\prime}$ were endpoints, $\left\{w \in U: u^{\wedge} 0 \subseteq w\right\}$ and $\left\{w \in U: u^{\wedge} 1 \subseteq w\right\}$ are also both densely ordered by $\leq_{\mathbb{Q}}$. Hence $u \in S^{\prime}$. By the same argument, $v \in S^{\prime}$ is found such that $s^{\wedge} 1 \subseteq v$. Note that $\left|I S\left(s, S^{\prime}\right)\right|=2$ for every $s \in S^{\prime}$. Let $f: S^{\prime} \rightarrow S$ be a fixed one-to-one function such that

$$
\text { a) } s^{\wedge} 0 \subseteq f(s)
$$

for every $s \in S^{\prime}$. It is easy to define such a function by induction because by the construction $\left\{s \in S: x^{\wedge} 0 \subseteq s\right\}$ is infinite for every $x \in S^{\prime}$. Let $Z$ be the perfect subtree of $S^{\prime}$ (see Figure 5) with the property that for every $x, y \in Z$ :

b) $|x|<|y|$ iff $|x|<|f(y)|$ iff $|f(x)|<|y|$ iff $|f(x)|<|f(y)|$,

c) if $x \in Z(m), y \in Z(n)$, then $|x|<|y|$ iff $m<n$ or $\left(m=n\right.$ and $\left.x<_{\text {lex }} y\right)$.

Again, it is easy to construct such a subtree because $S^{\prime}$ is perfect. Define by induction a bijection $\psi: W \rightarrow Z$ as follows: put $\psi(\operatorname{root}(W))=\operatorname{root}(Z)$. Having defined $\psi \uparrow \bigcup_{k \leq n} W(k)$, let $W(n+1)=\left\{w_{1}, \ldots, w_{2^{n+1}}\right\}$ and $Z(n+1)=\left\{z_{1}, \ldots, z_{2^{n+1}}\right\}$ be enumerations of $n+1$-st levels of $W$ and $Z$ respectively such that for all $1 \leq i, j \leq 2^{n+1}$ we have $i<j$ iff $\left|w_{i}\right|<\left|w_{j}\right|$ iff $\left|z_{i}\right|<\left|z_{j}\right|$. Then put $\psi\left(w_{i}\right)=z_{i}$ for all $1 \leq i \leq 2^{n+1}$. Note that we must have necessarily: 

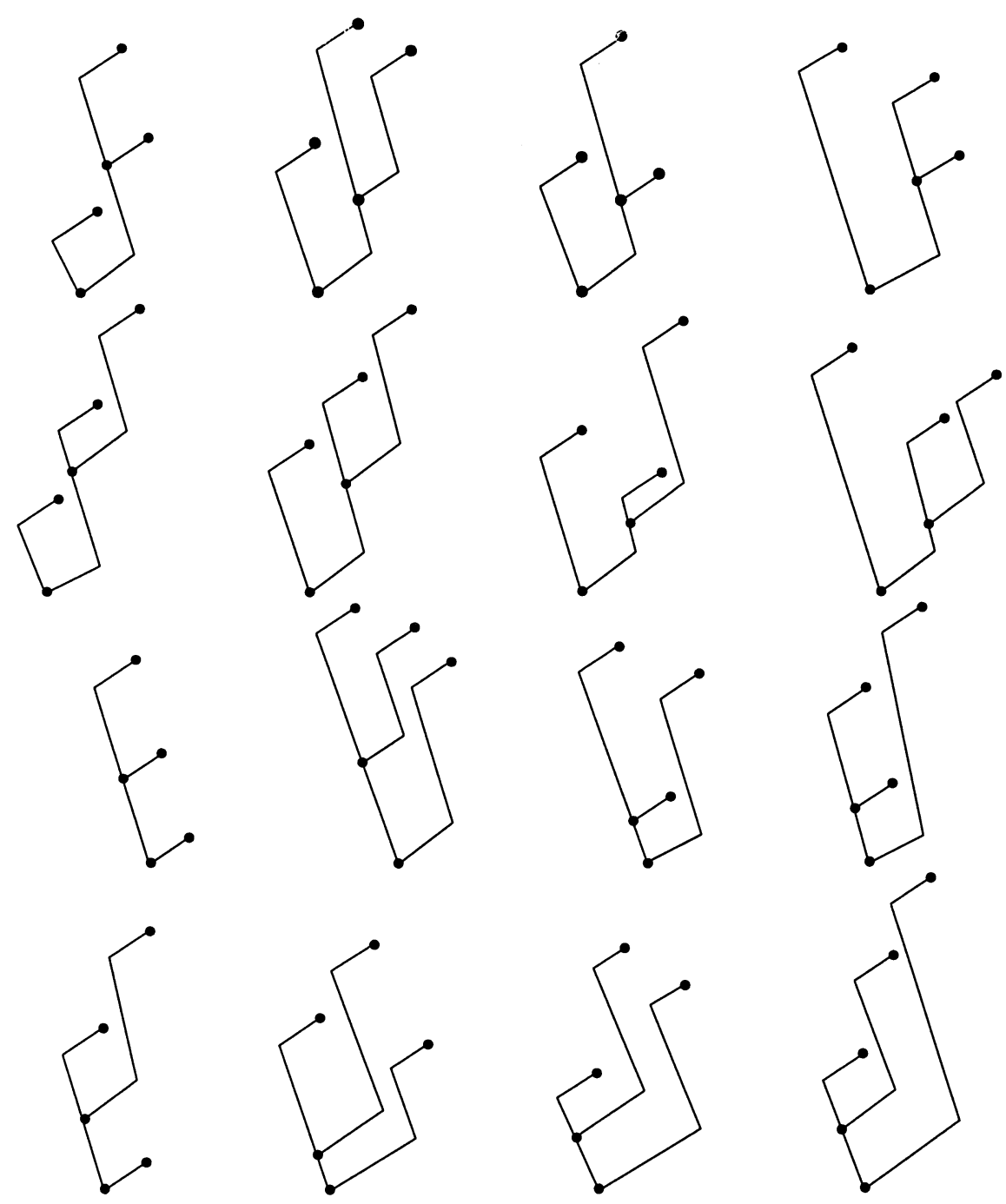

Figure 7. Embedding types in the case $n=3$

d) $w_{1} \wedge 0 \subseteq w_{2}$ iff $\psi\left(w_{1}\right)^{\wedge} 0 \subseteq \psi\left(w_{2}\right)$ for every $w_{1}, w_{2} \in W$ and

e) $\left(w_{1} \wedge w_{2}\right)^{\wedge} 0 \subseteq w_{1}$ iff $\left(\psi\left(w_{1}\right) \wedge \psi\left(w_{2}\right)\right)^{\wedge} 0 \subseteq \psi\left(w_{1}\right)$ for every $w_{1}, w_{2} \in W$.

Let $P=\left\{p_{1}, \ldots, p_{n}\right\}_{\leq_{Q}} \in[X]^{n}$. Let $P^{\prime}=\left\{p: p^{\wedge} 01 \in P\right\}, A^{\prime}=\psi^{\prime \prime} P^{\prime}$ and $A=f^{\prime \prime} A^{\prime}$. Let $f^{\prime}: \wedge(P) \rightarrow \wedge(A)$ be an extension of $f$ defined as follows: given $p_{i}, p_{i+1} \in P$ put $f^{\prime}\left(p_{i} \wedge p_{i+1}\right)=f\left(\psi\left(p_{i}^{\prime}\right)\right) \wedge f\left(\psi\left(p_{i+1}^{\prime}\right)\right)$ where $p_{i}^{\prime}, p_{i+1}^{\prime} \in P^{\prime}$ are unique elements such that $p_{i}^{\prime \wedge} 01=p_{i}$ and $p_{i+1}^{\prime}{ }^{\wedge} 01=p_{i+1}$, and for every $p_{i} \in P$ put $f^{\prime}\left(p_{i}\right)=f\left(\psi\left(p_{i}^{\prime}\right)\right)$ where $p_{i}^{\prime} \in P^{\prime}$ is the unique element such that $p_{i}^{\prime \wedge} 01=p_{i}$. It is easy to see that $f^{\prime}$ is a bijection from $\wedge(P)$ onto $\wedge(A)$. Let us prove that $f^{\prime}$ satisfies 1), 2) and 3) of Definition 0.4. 1) follows from the definition of $\psi$ and $f$ and $f^{\prime}$. By a), b) and c) we have that $f^{\prime}$ satisfies 2), and 3) follows from a), d) and e). Hence, $\wedge(P) \sim_{E m} \wedge(A)$. This finishes the proof of the lemma. 


\section{REFERENCES}

1. D.Devlin, Some partition theorems and ultrafilters on $\omega$, Ph.D. thesis, Dartmouth College (1979).

2. J.D.Halpern and H.Läuchli, A partition theorem, Trans. Amer. Math. Soc. 124 (1966), 360367. MR 34:71

3. K.Milliken, A Ramsey Theorem for Trees, J. Combinatorial Theory A 26 (1979), 215-237. MR 80j:05090

Department of Mathematics, University of Toronto, Toronto, Ontario, Canada MS5 $1 \mathrm{~A} 1$

E-mail address: voja@math.toronto.edu 